\title{
Microwave Extraction of Gallic Acid Equivalent of Total Flavonoids from Nigella sativa
}

\author{
Burcu OZTURK ${ }^{1}$, Sibel YIGITARSLAN*1,
}

${ }^{1}$ Department of Chemcial Engineering, Suleyman Demirel University, Isparta, Turkey

Received: 04 May 2017 - Revised: 23 June 2017 - Accepted: 05 August 2017

\begin{abstract}
Unfortunately, cancer is the most widespread disease in worldwide. Colorectal cancer is declared as the third most common cancer type. The usage of controlled-drug-releasing capsules for treating the cancer diseases has becoming more and more popular in the whole world. In the production of drug raw material, the first operation is an extraction of the phytochemicals from the herbal plant. The yield of the extraction operation is an important step for the economical and efficient production of such capsules. Thus the aim of this study was to optimize the microwave extraction of total flavonoids from Nigella sativa with methanol by using Response Surface Methodology based on Box-Behnken design. Microwave extraction experiments had been carried out with the parameters of microwave power, solid/liquid ratio and the and the application time. The optimum values were determined as $0.62 \mathrm{~kW}, 1 /(47) \mathrm{g} / \mathrm{mL}$, and 10.84 second, respectively. According to the quadratic surface of the extraction yield depending on those parameters, $309.60 \mathrm{mg} / 100 \mathrm{~g}$ of gallic acid equivalent of total flavonoids can be obtained at the optimum conditions. As a result of the statistical analysis, this function was found as capable of expressing the effect of the parameters on the amount of total flavonoid production in industry. Nowadays, drying of the extracts and loading of the aqueous solutions of them into the prebiotic-coated probiotic capsules have been under consideration.
\end{abstract}

Keywords: Nigella sativa, Extraction, Flavonoid, Response surface methodology

\section{INTRODUCTION}

In the past several years, polyphenols received considerable attention because of their contribution to the human health. Flavonoids are type of antioxidants and are member of polyphenols. They are also called as phenolic hydrogens because their hydrogen supplies electron to the free radicals resulting them to neutralize [1]. These bioactive compounds are capable of scavenging free radicals, avoiding lipid oxidation and reducing the formation and progression of certain types of cancers including breast, colon and prostate [2, 3]. Vegetables, fruits, herbs and cereals are main sources of polyphenols [4-6]. The essential oil of Nigella sativa was investigated and especially thymoquinone, carvacrol, $\mathrm{t}$-anethole and 4-terpineol components were determined as capable of radical scavenging activity [7]. Due to this antioxidant property, the herb is supposed to be used in cancer treatments since the antioxidant molecules inside have ability to bind the free radicals damaging the cell. Antioxidants also prevent DNA damaging due to lipid peroxidation resulting from the oxygen production of free

${ }^{*}$ Corresponding Author E-mail: yildizsibel@sdu.edu.tr 
radicals [8]. The crude extracts of the seeds of Nigella sativa have been reported as pharmacologically active; they protect against nephrotoxicity and hepatoxicity induced by either disease or chemicals, they also have antiinflammatory, analgesic, antipyretic, antimicrobial activity [9].

Solid-liquid extraction is a very important stage for later use of those bioactive compounds. The type and the amount of the flavonoids obtained from the herbs are strongly affected by the extraction. From the development of soxhlet extraction by F. Soxhlet in the nineteenth century, several different types of extraction techniques have been developed including ultrasonic, microwave, and supercritical [10-13]. Microwave extraction requires small amount of solvent and less extraction time, but the local temperature increases quickly due to the cavity effect. Thus, the optimization of the extraction conditions is inevitable.

Mathematical modeling of the extraction is a useful engineering tool which facilitates the understanding, optimization, design and control of the processes with minimal time and energy consumption. Todays the most approved model for the optimization, namely response surface method is a combination of statistical and mathematical techniques used for analyzing several independent variables and also interactive effects among the variables on the response. This method has been used in several optimizations including adsorption [14], extraction [15], fermentation [16], and production processes [17]. In addition, the final equation found by this method can be easily adaptable to any situation faced in the industrial scale production.

In the light of the literature cited above, the aim of the study was determined as optimization of microwave extraction of total flavonoids from Nigella sativa for its possible use in controlled drug releasing applications in cancer treatment. In order to investigate the combinational effects of the parameters, a software program called Design-Expert used with Response Surface Methodology due to the fact that this method has an advantage of prediction of the effects of several parameters simultaneously without doing any more experiment [1114]. Three-dimensional extraction surfaces are constructed by the software. The derivation of an industrially applicable equation representing the extraction surface of the quercetin equivalents of total flavonoids of the herb via microwave extraction was also realized.

\section{MATERIAL and METHODS}

Nigella sativa was purchased from a herbalist and the analytical grade chemicals (aluminum chloride, sodium acetate, and acetic acid) were purchased from Sigma Co. The experimental design optimization was applied in two steps; firstly single-parameter optimization was used by changing the range of the selected parameter while keeping all the others at their respective value, and then multiple-parameter was achieved with threeparameter-and-three-level Box-Behnken Design by using the results of the single-parameter optimization.

In order to study the effects of temperature, solid-to-liquid ratio, and time on the extracted amount of total flavonoids, independent variables were coded according to (1) in multipleparameter optimization studies:

$$
x_{i}=\frac{x_{i}-x_{0}}{\Delta x}
$$

where $x_{i}$ is the dimensionless coded value of $i$ th independent variable, $x_{0}$ is the value of $x_{i}$ at the center point, and $\Delta x$ is the step change value. The parameters of the study were summarized in Table 1 . The center points (coded as " 0 ") of the parameters were obtained from the single-parameter optimization results. The surface of the system is explained by the polynomial model given in (2) and higher orders:

$$
Y=\beta_{0}+\sum_{i=1}^{k} \beta_{i} x_{i}+\sum_{i=1}^{k} \beta_{i i} x_{i}^{2}+\sum_{i=1}^{k-1} \sum_{j=2}^{k} \beta_{i j} x_{i} x_{j}+\varepsilon
$$


where $Y$ is the predicted response, $x_{i}, x_{j}, \ldots, x_{k}$ are input variables, which affect the response $Y, x_{i}^{2}, x_{j}^{2}, \ldots, x_{k}^{2}$ are the square effects, $\beta_{0}$ is the intercept term, $x_{i} x_{j}, x_{j} x_{k}$ and $x_{i} x_{k}$ are the interaction effects, $\beta_{i}(i=1,2, \ldots, k)$ is the linear effect, $\beta_{i i}(i=1,2, \ldots, k)$ is the squared effect, $\beta_{i j}(i=1,2, \ldots, k)$ is the interaction effect, and $\varepsilon$ is the random error $[18,19]$.

Table 1. Box-Behnken Design parameters used in the study

\begin{tabular}{llll}
\hline Parameters & -1 & 0 & +1 \\
\hline $\mathrm{x}_{1}:$ Extraction time $(\mathrm{min})$ & 10 & 25 & 40 \\
$\mathrm{x}_{2}:$ Solid/liquid $(\mathrm{g} / \mathrm{ml})$ & $1 / 30$ & $1 / 45$ & $1 / 60$ \\
$\mathrm{x}_{3}:$ Microwave power $(\mathrm{kW})$ & 0.3 & 0.6 & 0.9 \\
\hline
\end{tabular}

The Design-Expert 9.0 (Stat-Ease Inc., Minneapolis, MN, USA) software was used for regression and graphical analysis of the experimental data to fit the equations developed and evaluation of their statistical significance. Box Behnken Design (BBD) is frequently used with response surface method due to its suitability to fit quadratic surface that usually works well for process optimization. The optimum values of the selected variables were obtained by solving the regression equation at desired values of the process responses was fixed at the optimization criteria.

Methanolic extraction was realized batch-wise in a $250 \mathrm{~mL}$ Erlenmeyer flask. Extraction time, solid-to-liquid ratio, and microwave power were chosen as the parameters of single- and multiple-parameter experimental designs. At the end of the specified extraction conditions the content of the flask was filtered through $110 \mathrm{~mm}$ filters (FilterLab) and filtered samples were used for total flavonoid analysis.

The concentrations of the total polyphenols in the extracts filtered were determined using the Folin-Ciocalteu method. In this paper, the main phenolics in Nigella sative were assumed as flavonoids, since they have the same effect on cancer disease treatments. In the analysis 0.4 $\mathrm{ml}$ of the extract was mixed with $5.1 \mathrm{ml}$ of distilled water and $0.5 \mathrm{ml}$ of Folin ciocalteu reagent. $1.5 \mathrm{ml}$ of sodium carbonate solution ( $20 \%$ by weight) was added into the medium immediately and after mixing they kept in dark during two hours at room temperature. The color resulted from the colorimetric reaction between gallic acid in the sample and the Folin reagent was analyzed by UV-vis spectrophotometer (Perkin-Elmer) at $765 \mathrm{~nm}$. The gallic acid equivalents (GAE) of total phenolics were calculated from the calibration curve (Absorbance $=0.01532 \mathrm{x}$ Concentration $\left.(\mu \mathrm{g} / \mathrm{ml}) ; \mathrm{R}^{2}=0.9989\right)$ and the results were expressed as mg GAE/g dry herb.

\section{RESULTS}

In the study, the parameters summarized in Table 1 was was used to construct the threelevel, three-parameter Box-Behnken design and fifteen experimental conditions were obtained. Microwave extractions were realized at each of the coded conditions and the coded parameters and their respective yields (as it was summarized in Table 2) were entered into the software. The Design-Expert software statistically analyzes all the experimental conditions and yields together and provides a comparison of the possible functions for the user. The user selects the most appropriate function expressing the response surface by comparing the statistical data and graphs of the functions contained in the program. The model having the highest regression coefficient and the lowest coefficient of variance value was chosen as the predicted best function for representation of the extraction surface. Those values for the suggested quadratic model of software were 0.9949 and 7.27, respectively. The predicted R-squared was found reasonably in agreement with the adjusted R-squared value of 0.9858 . Thus, the fitness in between the experimental data (actual) and their respective calculated values of the function 
(predicted) (Fig. 1) was approved the statistical conclusions. As a result, the model function was chosen as quadratic.

Table 2. Box-Behnken Design and experimental results

\begin{tabular}{cccccc}
\hline No & $\mathrm{x}_{1}$ & $\mathrm{x}_{2}$ & $\mathrm{x}_{3}$ & \multicolumn{1}{c}{ Abs } & GAE of total flavonoids $(\mathrm{mg})$ \\
\hline 1 & -1 & -1 & 0 & 0.3164 & 61.9376 \\
2 & +1 & -1 & 0 & 0.3845 & 75.2862 \\
3 & -1 & +1 & 0 & 0.4823 & 188.8524 \\
4 & +1 & +1 & 0 & 0.2160 & 84.5783 \\
5 & -1 & 0 & -1 & 0.1713 & 50.3167 \\
6 & +1 & 0 & -1 & 0.3168 & 93.0455 \\
7 & -1 & 0 & +1 & 0.7342 & 215.6593 \\
8 & +1 & 0 & +1 & 0.3041 & 89.3155 \\
9 & 0 & -1 & -1 & 0.3100 & 60.6990 \\
10 & 0 & +1 & -1 & 0.1521 & 59.5513 \\
11 & 0 & -1 & +1 & 0.4195 & 82.1229 \\
12 & 0 & +1 & +1 & 0.6135 & 240.2501 \\
13 & 0 & 0 & 0 & 0.2642 & 67.6044 \\
14 & 0 & 0 & 0 & 0.2288 & 45.1861 \\
15 & 0 & 0 & 0 & 0.1644 & \\
\hline
\end{tabular}

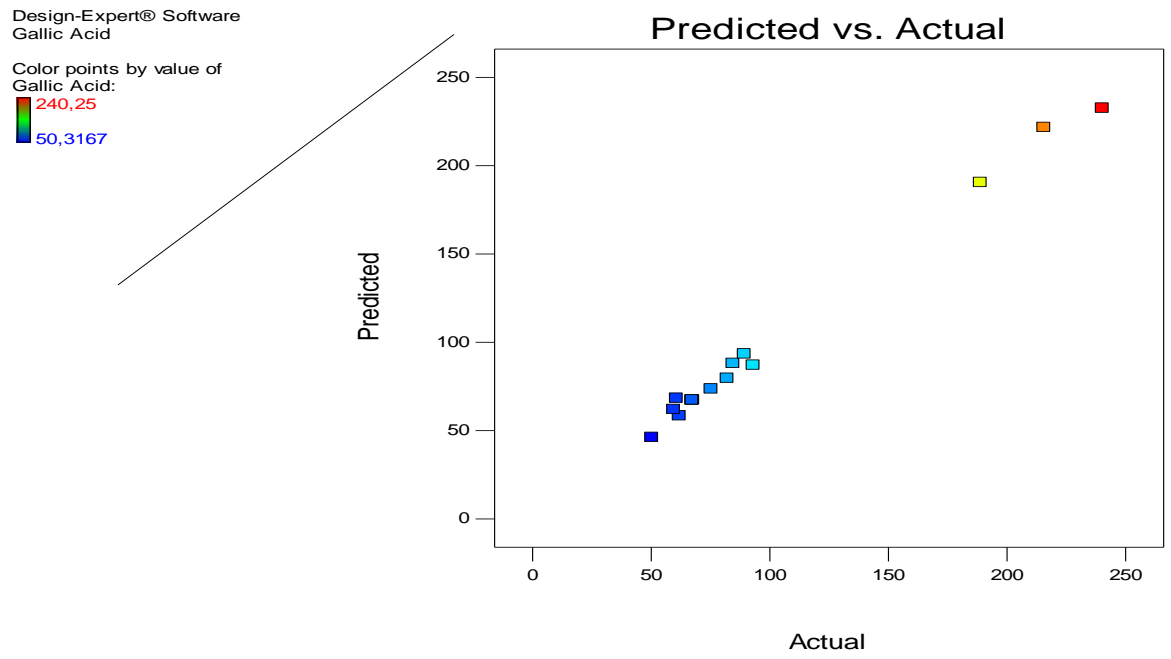

Figure 1. Statistical approval of the reduced cubic model.

The Analysis of Variance (ANOVA) for the quadratic model was constructed by the software (Table 3). In that, A demonstrates the time; $\mathrm{B}$ and $\mathrm{C}$ were representing solid-to-liquid ratio and microwave power, respectively. Since the larger the magnitude of the F-value and the smaller the p-value, the more significant the corresponding coefficient. The fact that the selected model is "significant" and "lack of fit" is "not significant" (Table 3) is the main reason for choosing the best expressing function as "quadratic". The most effective single parameters on the extraction of gallic acid equivalent of total flavonoids from Nigella sativa were found as microwave power followed by solid-to-liquid ratio. Although the extraction time seemed to be the least effective single parameter, the interrelation between time and other parameters selected were found considerable. 
Table 3. Analysis of variance (ANOVA) for quadratic function

\begin{tabular}{lccccc}
\hline Source & $\begin{array}{c}\text { Sum of } \\
\text { Squares }\end{array}$ & df & $\begin{array}{c}\text { Mean } \\
\text { Square }\end{array}$ & $\begin{array}{c}\text { F } \\
\text { Value }\end{array}$ & $\begin{array}{c}\text { p-value } \\
\text { Prob }>\text { F }\end{array}$ \\
\hline Model & 52297,92 & 9 & 5810,88 & 109,29 & $<0.0001$ \\
A-Time & 3808,05 & 1 & 3808,05 & 71,62 & 0,0004 \\
B-S/L & 10744,78 & 1 & 10744,78 & 202,09 & $<0.0001$ \\
C-M.Power & 16537,92 & 1 & 16537,92 & 311,05 & $<0.0001$ \\
AB & 3458,77 & 1 & 3458,77 & 65,05 & 0,0005 \\
AC & 7146,39 & 1 & 7146,39 & 134,41 & $<0.0001$ \\
BC & 6342,12 & 1 & 6342,12 & 119,28 & 0,0001 \\
A2 & 1244,77 & 1 & 1244,77 & 23,41 & 0,0047 \\
B2 & 1058,62 & 1 & 1058,62 & 19,91 & 0,0066 \\
C2 & 2564,27 & 1 & 2564,27 & 48,23 & 0,0010 \\
Residual & 265,84 & 5 & 53,17 & & \\
Lack of Fit & 265,75 & 3 & 88,58 & 1941,08 & 0,0005 \\
Pure Error & 0,091 & 2 & 0,046 & & \\
Cor Total & 52563,76 & 14 & & & \\
\hline
\end{tabular}

Model: Significant; Lack of fit: Not significant; R-Squared: 0.9949; Adjusted R-Squared: 0.9858; Predicted RSquared: 0.9191

At this point, three-dimensional response surfaces were constructed in this study by using software. The interactive effects of the parameters were shown in Figures 2-4. In those, red regions shows the highest amount of total flavonoids extracted, yellow and blue parts represent the lower and much lower extraction yields than those. As it can be seen from figures, solid-toliquid ratio and microwave combinations can produce the highest yield, and neither microwave power-and-time nor solid-to-liquid ratio-time combinations can reach it. In order to reach the highest flavonoid amount, both the solid-to-liquid ratio and the temperature must be in the coded range of $[0.5 ; 1]$ (Figure 3 ).

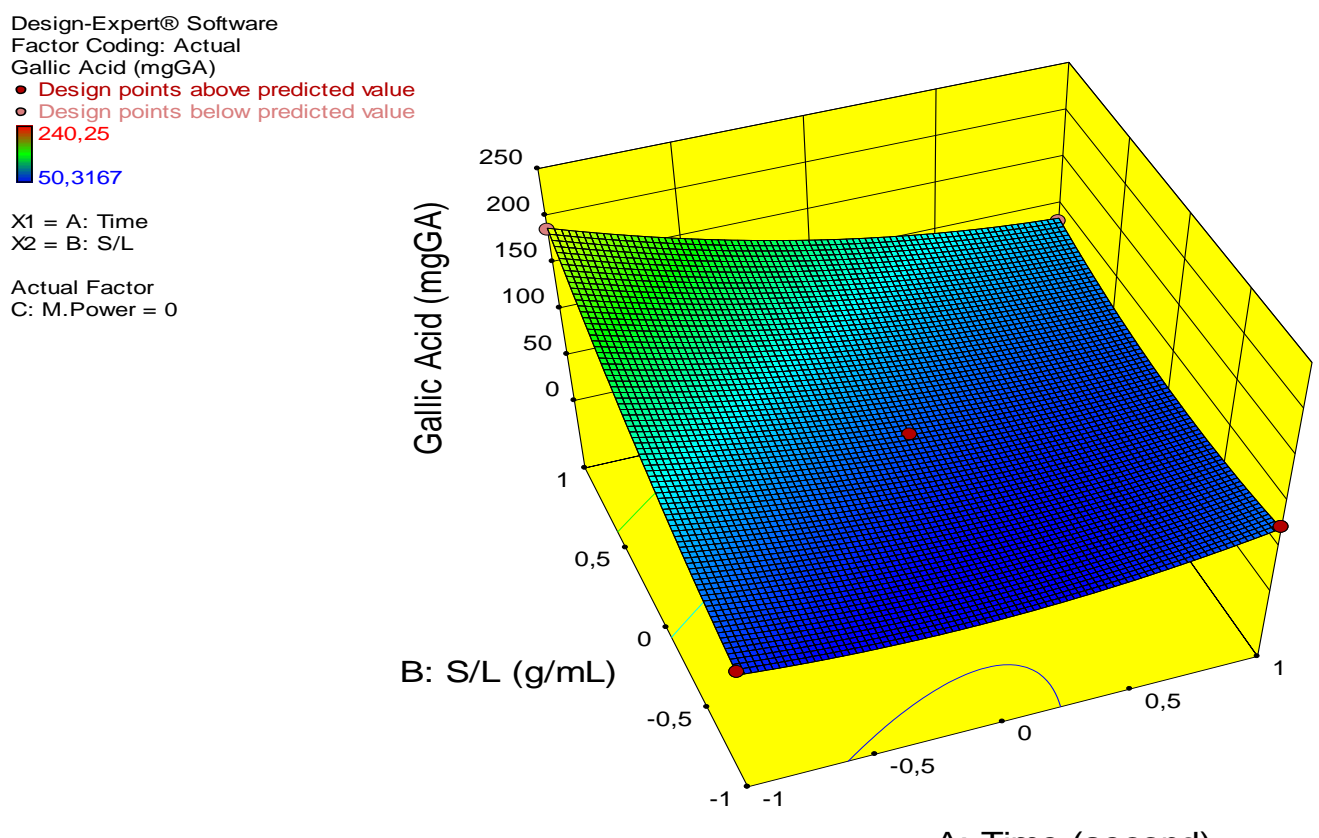

A: Time (second)

Figure 2. Three-dimensional response surface of extraction yield depending on solid-to-liquid ratio and extraction time. 
Design-Expert ${ }^{\circledR}$ Software

Factor Coding: Actual

Gallic Acid (mgGA)

- Design points above predicted value

- Design points below predicted value

240,25

50,3167

$\mathrm{X} 1=\mathrm{C}:$ M.Power

$X 2=B: S / L$

Actual Factor

A: Time $=0$

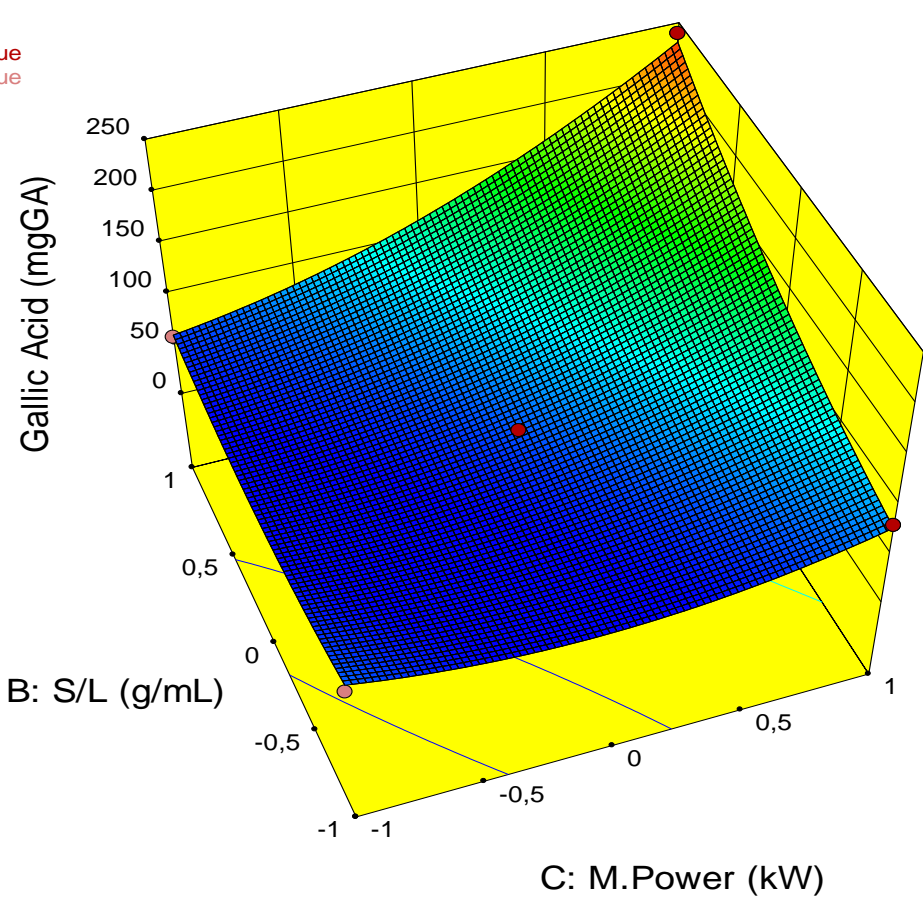

Figure 3. Three-dimensional response surface of extraction yield depending on solid-to-liquid ratio and microwave power.

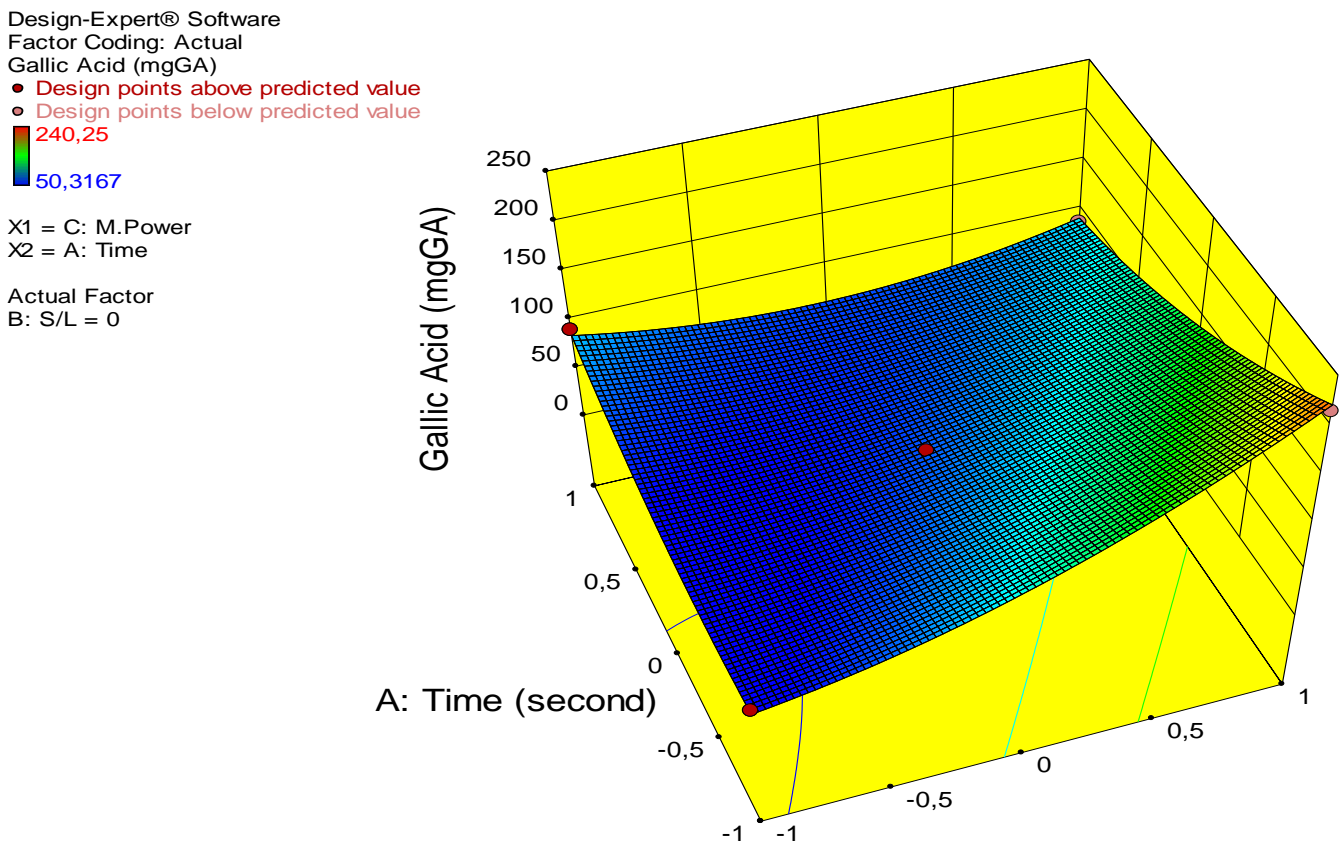

C: M.Power (kW)

Figure 4. Three-dimensional response surface of extraction yield depending on extraction time and microwave power.

Here we demonstrated that $O$. basilicum effect differently on BM- and DP- mesenchymal stem cells. It is important to determine the differentiation, and proliferation of cells with promising inductive agents. Studies should avoid to suggest any plant extract as an agent without evidence of safe. 


\section{DISCUSSION and CONCLUSION}

In this study our aim was to optimize the microwave extraction conditions of gallic acid equivalent of total flavonoids from Nigella sativa by investigating the effect of parameters for each (microwave power, extraction time and solid-to-liquid ratio) and also in combination. In addition, in order to use the results of the study in an industrial production processes, modeling equation was derived. Quadratic function (Eqn. 4) was found as the best function representing the extraction surface and all the statistical analysis approved this conclusion:

$$
\begin{aligned}
& \text { Gallic acid equivalent of total flavonoids }=67.37-(21.82) A+(36.65) B+ \\
& (45.47) C-(29.41) A B-(42.27) A C+(39.82) B C+(18.36) A^{2}+(16.93) B^{2}+ \\
& (26.35) C^{2}
\end{aligned}
$$

The most and the least effective parameters of the extraction were determined as microwave power, and the application time, respectively. Finally, the required extraction conditions were analyzed by using this equation and numerical analysis section of the software. In the analysis, restrictions of the parameters were selected as "in range" (has a meaning that they are in the experimental range), and the response criterion was determined as "max". As a result of the multi-parameter optimization, the optimum conditions producing the highest yield (309.60 mg/100g of gallic acid equaivalent of total flavonoids) were determined as $0.62 \mathrm{~kW}$, 10.84 seconds, $1 / 47 \mathrm{~g} / \mathrm{mL}$. Nowadays, drying of the extracts and loading of the aqueous solutions of them into the prebiotic-coated probiotic capsules have been under consideration.

\section{Conflict of Interests}

Authors declare that there is no conflict of interests.

\section{REFERENCES}

[1] Rice-Evans, C. A., Miller, N. J., \& Paganga, G. (1996). Structure-antioxidant activity relationships of flavonoids and phenolic acids. Free radical biology and medicine, 20(7), 933-956.

[2] Sauvaget, C., Nagano, J., Hayashi, M., Spencer, E., Shimizu, Y., \& Allen, N. (2003). Vegetables and fruit intake and cancer mortality in the Hiroshima/Nagasaki Life Span Study. British journal of cancer, 88(5), 689-694.

[3] Scalbert, A., Johnson, I. T., \& Saltmarsh, M. (2005). Polyphenols: antioxidants and beyond. The American journal of clinical nutrition, 81(1), 215S-217S.

[4] Sant'Anna, V., Brandelli, A., Marczak, L. D. F., \& Tessaro, I. C. (2012). Kinetic modeling of total polyphenol extraction from grape marc and characterization of the extracts. Separation and purification technology, 100, 82-87.

[5] Minozzo, M., Popiolski, A., Dal Prá, V., Treichel, H., Cansian, R. L., Oliveira, J. V., ... \& Mazutti, M. A. (2012). Modeling of the overal kinetic extraction from Maytenus aquifolia using compressed CO2. Brazilian Journal of Chemical Engineering, 29(4), 835-843.

[6] Jokić, S., Velić, D., Bilić, M., BuCić-koJić, A., PlANiNić, M., \& ToMAS, S. (2010). Modelling of the process of solid-liquid extraction of total polyphenols from soybeans. Czech Journal of Food Sciences, 28(3), 206-212.

[7] Burits, M., \& Bucar, F. (2000). Antioxidant activity of Nigella sativa essential oil. Phytotherapy research, 14(5), 323-328.

[8] Ait Mbarek, L., Ait Mouse, H., Elabbadi, N., Bensalah, M., Gamouh, A., Aboufatima, R., ... \& Zyad, A. (2007). Anti-tumor properties of blackseed (Nigella sativa L.) extracts. Brazilian Journal of Medical and Biological Research, 40(6), 839-847. 
[9] Ali, B. H., \& Blunden, G. (2003). Pharmacological and toxicological properties of Nigella sativa. Phytotherapy Research, 17(4), 299-305.

[10] Wan, H. B., \& Wong, M. K. (1996). Minimization of solvent consumption in pesticide residue analysis. Journal of Chromatography A, 754(1-2), 43-47.

[11] Eskilsson, C. S., \& Björklund, E. (2000). Analytical-scale microwave-assisted extraction. Journal of Chromatography A, 902(1), 227-250.

[12] Lapornik, B., Prošek, M., \& Wondra, A. G. (2005). Comparison of extracts prepared from plant by-products using different solvents and extraction time. Journal of food engineering, 71(2), 214-222.

[13] Zancan, K. C., Marques, M. O., Petenate, A. J., \& Meireles, M. A. A. (2002). Extraction of ginger (Zingiber officinale Roscoe) oleoresin with CO 2 and co-solvents: a study of the antioxidant action of the extracts. The Journal of supercritical fluids, 24(1), 57-76.

[14] Turkyilmaz, H., Kartal, T., \& Yildiz, S. Y. (2014). Optimization of lead adsorption of mordenite by response surface methodology: characterization and modification. Journal of Environmental Health Science and Engineering, 12(1), 5.

[15] Goktas, F. M., Sahin, B., \& Yigitarslan, S. (2015). Production of sterilizing agents from Calendula officinalis extracts optimized by response surface methodology. International journal of analytical chemistry, 2015.

[16] Dashtianeh, M., Vatanara, A., Fatemi, S., \& Sefidkon, F. (2013). Optimization of supercritical extraction of Pimpinella affinis Ledeb. using response surface methodology. Journal of CO2 Utilization, 3, 1-6.

[17] Levin, L., Herrmann, C., \& Papinutti, V. L. (2008). Optimization of lignocellulolytic enzyme production by the white-rot fungus Trametes trogii in solid-state fermentation using response surface methodology. Biochemical Engineering Journal, 39(1), 207-214.

[18] Zhao, Y., Hou, Y., Tang, G., Cai, E., Liu, S., Yang, H., ... \& Wang, S. (2014). Optimization of ultrasonic extraction of phenolic compounds from Epimedium brevicornum maxim using response surface methodology and evaluation of its antioxidant activities in vitro. Journal of analytical methods in chemistry, 2014.

[19] Sun, Z., Su, R., Qiao, J., Zhao, Z., \& Wang, X. (2014). Flavonoids extraction from Taraxacum officinale (Dandelion): optimisation using response surface methodology and antioxidant activity. Journal of Chemistry, 2014. 